\title{
Dye Release Experiments with Dextran Loaded Vesicles
}

Marc-Antoine Sani ${ }^{1^{*}}$, Neil O'Brien-Simpson ${ }^{2}$ and Frances Separovic ${ }^{1}$

${ }^{1}$ School of Chemistry, The University of Melbourne, Melbourne, Australia; ${ }^{2}$ Melbourne Dental School, The University of Melbourne, Melbourne, Australia

*For correspondence: msani@unimelb.edu.au

[Abstract] Dye release experiments are a widely used method to assess the interactions between membrane-active molecules and lipid membranes. Of particular interest is the ability to assess the degree of the lipid bilayer perturbation by simultaneously encapsulating dye of different sizes, such as dextrans grafted with a chromophore. In this assay, dextran linked to rhodamine or fluorescein are both encapsulated in lipid vesicles to allow quantifying the leakage of each dextran individually from a single sample. For instance, the size evaluation of the lipid pore formed by an antimicrobial peptide has been recently achieved using this protocol (Sani et al., 2013).

\section{Materials and Reagents}

1. Rhodamine-dextran (RD) of different molecular weights [for instance $40 \mathrm{kDa} R D$ molecular weight (RD-40)]

2. Fluorescein-dextran (FD) of different molecular weights [for instance 4.4 kDa FD (FD-4)]

3. Tris $\cdot \mathrm{HCl}$

4. $\mathrm{NaCl}$

5. MQ-water

6. Triton-X100

7. Peptide of interest e.g. Maculatin 1.1 (Sani et al., 2013)

8. Lipids of interest e.g. 1-palmitoyl-2-oleoyl-sn-glycero-3-phosphocholine (POPC), 1palmitoyl-2-oleoyl-sn-glycero-3-phosphoethanolamine (POPE), 1-palmitoyl-2-oleoyl-snglycero-3-phosphoglycerol (POPG), 1',3'-bis[1,2-dioleoyl-sn-glycero-3-phospho]-snglycerol (TOCL) (Sani et al., 2013)

\section{Equipment}

1. Polycarbonate membrane filters (200 $\mathrm{nm}$ diameter)

2. Extruder (for instance Avanti Mini-extruder, Alabaster)

3. Bench centrifuge 
4. Spectrofluorimeter and quartz cuvette

\section{Procedure}

A. Stock preparation

1. Prepare a Tris $\mathrm{HCl}+\mathrm{X} \mathrm{NaCl}$ buffer (buffer $A$ ) and a Tris $\mathrm{HCl}+(X-$ Dextran concentration) $\mathrm{NaCl}$ buffer (buffer $\mathrm{B}$ ) at desired $\mathrm{pH}$, with $\mathrm{X}$ being the $\mathrm{NaCl}$ concentration.

2. Prepare a peptide stock solution (The concentration will depend on the peptide solubility and the required lipid to peptide molar ratio under investigation.) in buffer A. Glass vials are recommended.

3. Prepare a stock solution of equimolar RD and FD in buffer $B$.

4. Co-solubilize lipids in chloroform/methanol (3:1 v/v), remove the organic solvents under vacuum using a rotary evaporator. Rehydrate the lipids in Milli-Q water and lyophilize.

5. Resuspend the require mass of fluffy lipid powder with the dextran stock solution. A 15 $\mathrm{mM}$ lipid stock solution usually allows practical dilution to reach required lipid to peptide molar ratio, but will be dependent on the peptide stock concentration. Perform 3-5 freezethaw cycles to homogenise the liposome dispersion, usually without a time delay between cycles, by dipping the solution in liquid nitrogen and then melting above the lipid fluid phase transition for $\sim 5$ min.

6. Extrude 10 times through an Extruder using $0.2 \mathrm{~mm}$ pore size polycarbonate filters to produce large unilamellar vesicles (LUV) of $200 \mathrm{~nm}$ diameter. Extrusion must be performed above the gel-to-fluid lipid phase transition to avoid vesicle aggregation or demixing of heterogeneous lipid compositions, if used.

7. Remove un-encapsulated dye from LUV dispersions by centrifuging three times at 22,000 $x g$ for $30 \mathrm{~min}$ at $25{ }^{\circ} \mathrm{C}$. After each centrifugation, remove the supernatant and replace with an equal volume of fresh buffer $B$ solution and mixed gently.

8. Determine the lipid concentration of washed LUV dispersions in triplicate using the phosphorus assay of Sani et al. (2013).

B. Sample preparation

1. Equilibrate the LUV dispersion and peptide solution at the desired temperature.

2. Depending on fluorimeter performance, prepare an adequate dextran-encapsulated solution, $\sim 200 \mu \mathrm{M}$ gives a high signal in our experience.

3. Add the require amount of peptide to obtain the lipid to peptide molar ratio (L/P) of interest. For reproducibility and statistical analysis, it is best to prepare the sample in triplicate. 
4. Produce negative control by adding buffer $B$ instead of the peptide solution. A nonmembrane active peptide or protein, such as bovine serum albumim, can also be used.

5. Produce positive control by adding $0.5 \%$ Triton X-100 (TX).

6. Incubate the samples for $30 \mathrm{~min}$ at the desired temperature.

7. Centrifuge the samples at $22,000 \times g$ for $30 \mathrm{~min}$.

8. Recover the supernatant, with care to leave the pellet unperturbed. Usually, $500 \mu \mathrm{l}$ sample are prepared and $300 \mu \mathrm{l}$ recovered for analysis.

9. Transfer the supernatant to a quartz cuvette.

C. Fluorescence measurement

1. Record the fluorescence emission of RD (then FD) using an excitation wavelength set at $550 \mathrm{~nm}$ (then $480 \mathrm{~nm}$ ). Fluorescence emission is recorded from 560 to $650 \mathrm{~nm}$ using adequate integration time and PMT voltage. Triplicate the measurements.

2. The amount of fluorescence is determined by integrating the area under the emission curve. The \% release of each sample is calculated by using the following equation: $\%_{\text {Dye Release }}=100 \times\left(I-I_{0}\right) /\left(I_{\max }-I_{0}\right)_{\text {where, }} \mathrm{I}$ is the fluorescence intensity of peptide treated vesicle supernatant, Io obtained from the peptide-free sample (negative control) supernatant, and Imax from the Tx treated supernatant (positive control).

\section{Acknowledgments}

None.

\section{References}

1. Anderson, R. L. and Davis, S. (1982). An organic phosphorus assay which avoids the use of hazardous perchloric acid. Clinica Chimica Acta 121(1): 111-116.

2. Sani, M. A., Whitwell, T. C., Gehman, J. D., Robins-Browne, R. M., Pantarat, N., Attard, T. J., Reynolds, E. C., O'Brien-Simpson, N. M. and Separovic, F. (2013). Maculatin 1.1 disrupts Staphylococcus aureus lipid membranes via a pore mechanism. Antimicrob Agents Chemother 57(8): 3593-3600. 